\title{
MANAGEMENT OF ZYGOMATICO-ORBITAL FRACTURES USING RIGID INTERNAL FIXATION WITH COSMETIC SURGICAL CONSIDERATIONS - CASE REPORT
}

Ong AHM. Management of zygomatico-orbital fracturers using rigid internal fixation with cosmetic surgical considerations - case report. Annal Dent. Univ Malaya 1996; 3: 51-55

\begin{abstract}
The current standard of managing facial bone fractures is the use of rigid internal fixation. This method provides good stabilization and repair for mid-face fractures such as the zygomatico-orbital fracture. Nowadays, for the young and old, patients want not only rapid bone healing, but also good facial aesthetics after surgical treatment following maxillofacial trauma. Therefore, osteosynthesis of fractures and inconspicuous post-operative scars are considered essential. The lower eyelid approach or modified blepharoplasty provides rapid access to the infraorbital rim as well as the orbital floor, while the lateral eyebrow incision gives direct access for fixation at the fronto-zygomatic suture. Combining the Gillies' approach and a single form of rigid internal fixation, good cosmetic results and function can be achieved. Cases using the micro-plate-system for thin infraorbital bones and the miniplate-system for thicker facial bones involving zygomatico-orbital fractures are described.
\end{abstract}

Key Words: Zygomatico-orbital fractures, rigid internal fixation, osteosynthesis plates, cosmetic surgery

\section{INTRODUCTION}

Many approaches or techniques are used in the management of zygomatico-orbital fractures. With surgical intervention, a simple elevation via the Gillies' or temporal fossa approach is adequate and effective in treating the majority of these fractures. Ellis et al. reported that only $30 \%$ of the surgical cases needed fixation of the reduced bone(s) (1). In the past, wires were commonly used to immobilise unstable fractures. However, the current practice is to use rigid internal fixation when managing the maxillo-facial fractures as plating provides better stabilisation and repair. The advantages of plating osteosynthesis compared with wire osteosynthesis have well been documented by Jones et al. (2), Michelet et al. (3) and Marsh (4). Besides being beneficial, it was shown by Thaller et al. that plating systems used on other fractures were cost effective as well (5).

Mini-plate system is used to immobilise frontozygomatic fractures via the lateral brow incisions. Studies done by $\mathrm{Bahr}$ and his co-worker reported that the 1.5 $\mathrm{mm}$ screw system could be used instead of the $2.0 \mathrm{~mm}$ system even in the mid-face regions associated with high functional loads (6). When the facial bone fractured is thin, for example at the infraorbital rim, the use of the micro-plate-system is indicated for good cosmetic results and minimal interference with the overlying soft tissues (7). The development of these smaller screw systems such as the $0.8,1.0,1.2$, and $1.4 \mathrm{~mm}$ systems are meant for use in low stress areas. Internal fixation at the infraorbital rim as well as exploration of the floor of the orbit can be done via the lower eyelid incision or modified blepharoplasty as this gives a rapid approach
AHM Ong BDS(S'pore), MSc(Lond)*

* Lecturer,

Department of Oral and Maxillofacial Surgery,

Faculty of Dentistry,

University of Malaya,

50603 Kuala Lumpur.

Malaysia. to the site of fracture $(8,9)$. In this paper, two clinical cases of patients who had sustained zygomatico-orbital fracture and treated using rigid internal fixation with cosmetic surgical considerations, are presented.

\section{CASE REPORTS}

\section{Case 1}

A 45-year-old male Chinese was first seen at the Accident and Emergency Unit, University Hospital, Kuala Lumpur for an alleged road traffic accident. He was knocked down by a motorcyclist while crossing the road and was rendered unconscious. At the time of admission, he was conscious but confused with Glasgow Coma Scale (GCS) of 9/15 (Eyes - 1, Verbal - 3, Motor - 5). There was epistaxis and cerebrospinal fluid (CSF) rhinorrhea from his left nostril. He had no

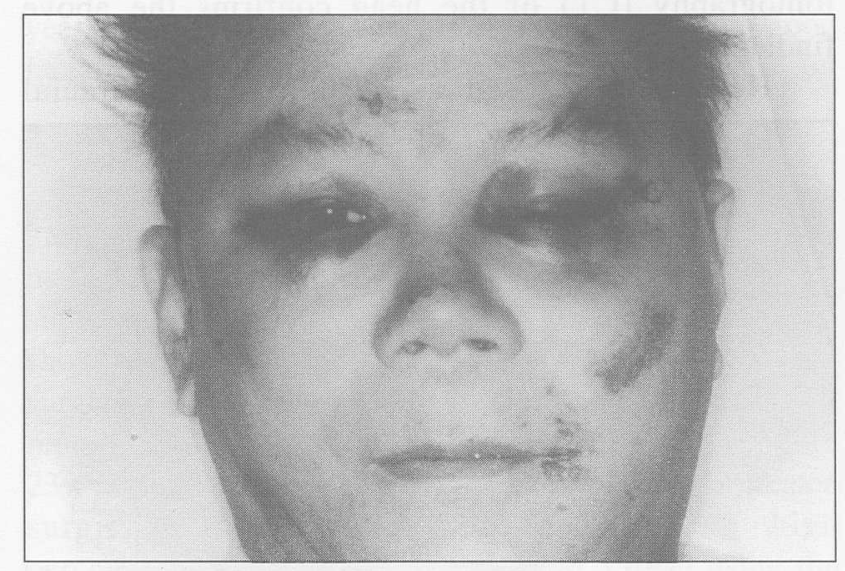

Figure 1. Clinical presentation showing facial laceration, abrasions, bilateral periorbital oedema and circumorbital ecchymosis. 
relevant past medical history. When neurologically stable with the GCS of $14 / 15$, he was referred to the Opthalmologist Unit for the visual impairment of his left eye and the Oral and Maxillofacial Unit for further management of the facial fractures.

An ophthalmic examination showed no light perception on the left eye which was swollen and unable to open. The right pupil was $5 \mathrm{~mm}$ and reactive but the left pupil was dilated and fixed. The loss of vision of the left eye was diagnosed as probably due to traumatic optic nerve injury. No active treatment was recommended.

Clinical examination of the maxillofacial region revealed a $5 \mathrm{~mm}$ laceration of the left frontal area, superficial facial abrasions, bilateral circumorbital ecchymosis and periorbital oedema (Fig. 1). There was subconjunctival haemorrhage but no light perception on the left eye. The left zygomatic bone was depressed. No nasal discharge or bleeding was observed and the maxilla was stable. The patient was able to open his mouth for about $2 \mathrm{~cm}$ interincisally and there was limitation of mandibular movement. Intraorally, the occlusion was

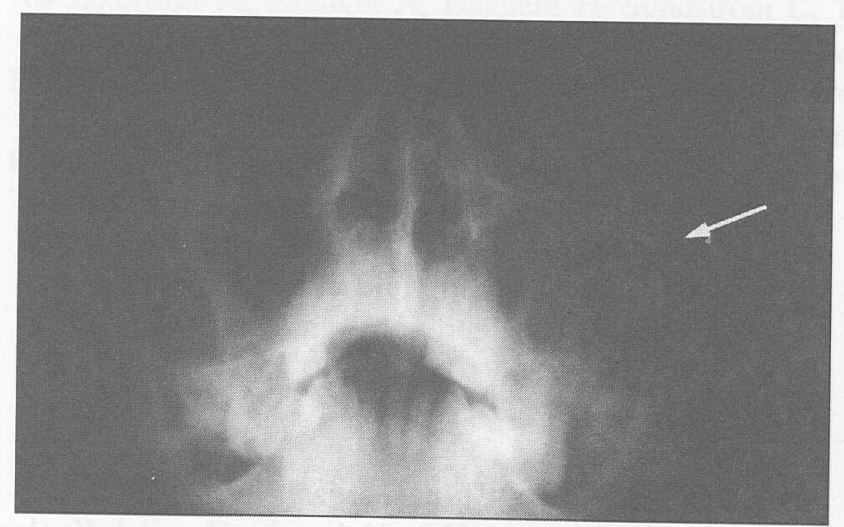

Figure 2. Occipitomental radiograph showing the left depressed zygomatico-orbital fracture.

satisfactory and the teeth present were not fractured.

Radiologically, a left coronoid fracture was evident in the orthopantomogram. The occipito-mental view showed fracture of the left malar bone (Fig. 2) and the frontal bone fracture was comminuted. Computed tomography (CT) of the head confirms the above findings.

He was diagnosed as sustaining a panfacial

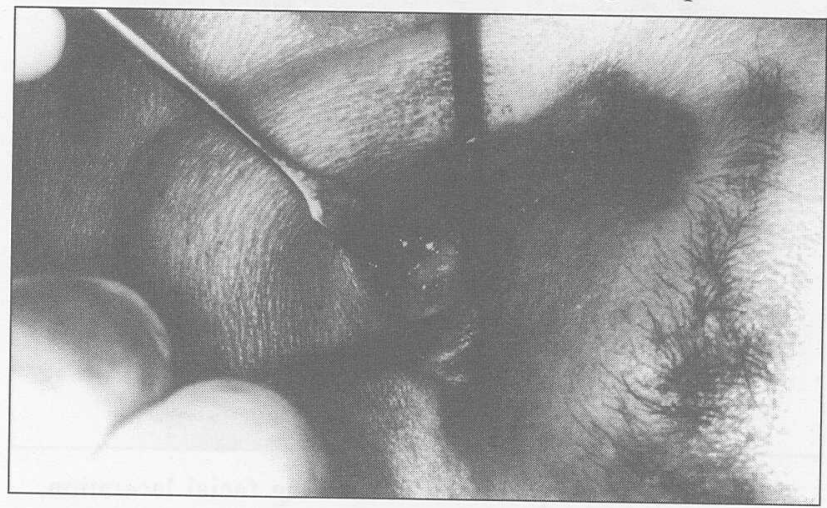

Figure 3. Modified blepharoplasty approach showing the micro-plate at the left infraorbital rim. fracture involving the comminuted fracture of left frontal bone, fronto-temporal bone, malar bone with the associated optic nerve injury and fracture left coronoid of the mandible.

The treatment plan was to elevate the left zygomatic complex via the Gillies' approach, exploration of the floor of the left orbit via the lower eyelid incision and fixation of the infraorbital rim. At operation, he underwent an open reduction to align the fractured zygomatic bone and fixation with the micro-platesystem at the infraorbital rim (Fig. 3). To achieve good cosmetic results the lower eyelid or blepharoplasty postorbicularis approach as described by Lacy and Pospisil (9) was performed. This consisted of a skin incision made about 2-3 $\mathrm{mm}$ below the grey line of the lower eyelid running parallel and along the whole length of its margin. The extended lateral incision was delineated but not incised. The orbicularis muscle was divided below the incision line and the dissection was continued in a downward direction in the plane posterior to the orbicularis muscle but anterior to the orbital septum. The inferior orbital margin was exposed after incision of the periosteum, which was carried out a few millimetres below the orbital rim to permit good closure of the periorbita after bony repair. Medially, the periosteum was elevated with great care, to avoid damage to the inferior oblique muscle. The floor of the orbit was explored and found to be intact without entrapment of tissue, displacement or communication with

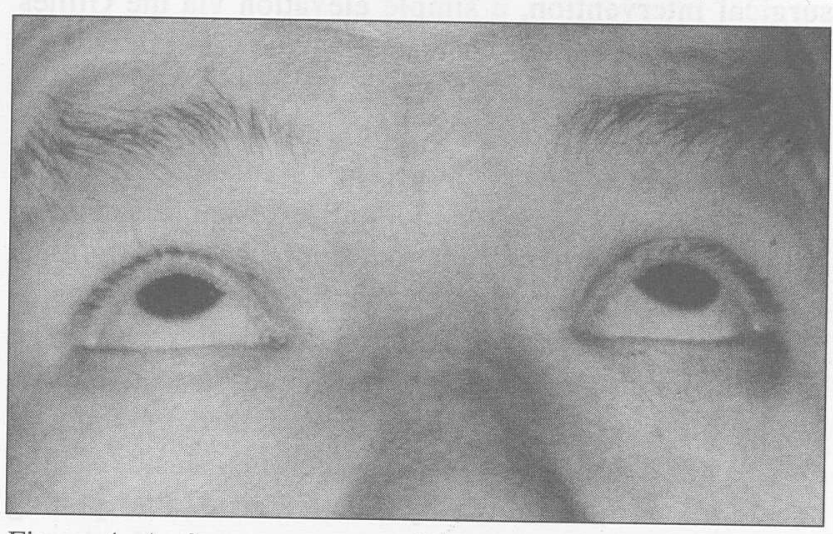

Figure 4. A. Scar appearance following the left lower eyelid or modified blepharoplasty incision.

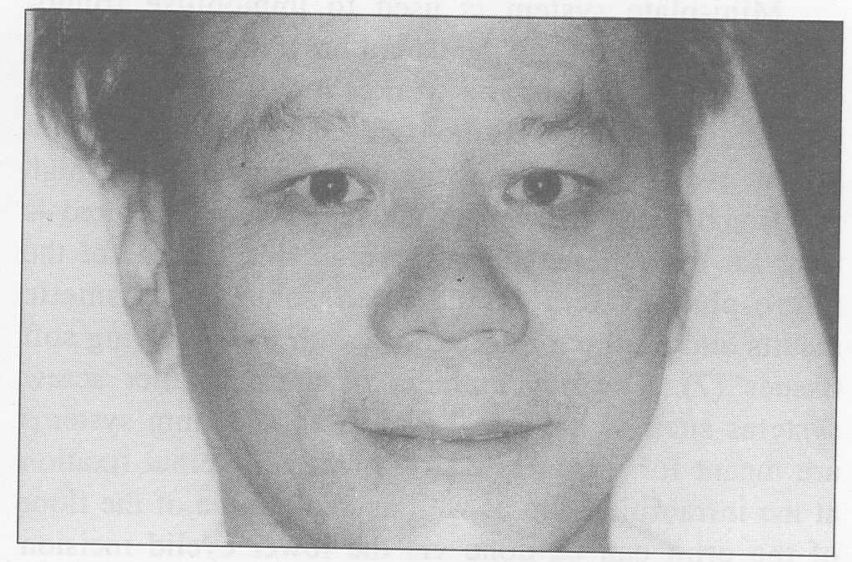

Figure 4. B. Postoperative appearance six weeks later 
the antrum. The fracture was stable following good alignment and a single fixation. A postoperative Frost suture accompanied by periorbital pressure dressing was used to reduce the incidence of ectropion.

Postoperative healing was satisfactory. His CSF rhinorrhoea resolved spontaneously and he recovered from his close head injury. Review of the patient after six weeks showed the mouth opening and mandibular movements were normal but he remained blind in his left eye (Fig. 4A, 4B). Two years later, the patient was still symptom free with good facial aesthetics and occlusal function.

\section{Case 2}

A 22 year-old Chinese man was brought to the University Hospital, Kuala Lumpur, following a road traffic accident. He skidded on his motorbike and was rendered unconscious. Initial treatment when admitted to the Accident and Emergency Department, included placing upper and lower arch bar to stop bleeding from the fractured sites. He was then referred to the Oral and Maxillofacial Department for further treatment when

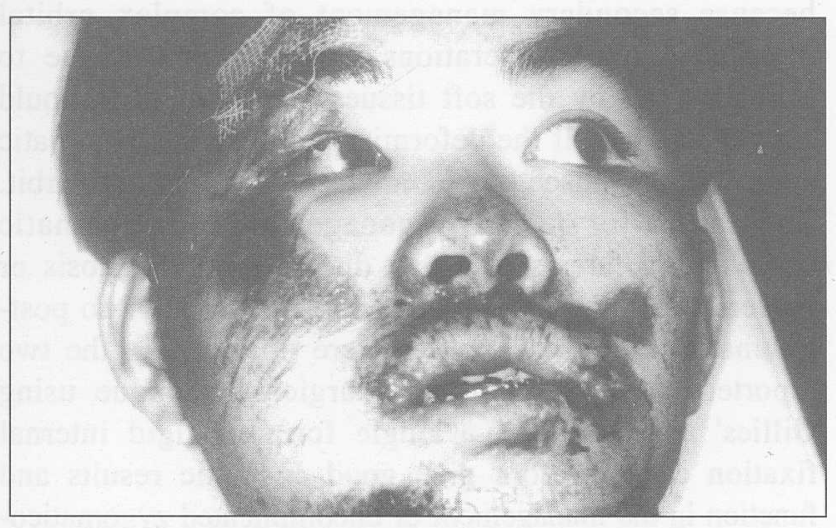

Figure 5. Clinical presentation showing facial laceration, abrasions and the swollen right upper eyelid.

neurologically stable.

On examination he was found to have a right supraorbital laceration and swollen upper eyelid as well as superficial abrasions on the cheeks, lips and chin (Fig. 5). No diplopia or nasal discharge was observed and the mouth opening was limited to about $3 \mathrm{~cm}$

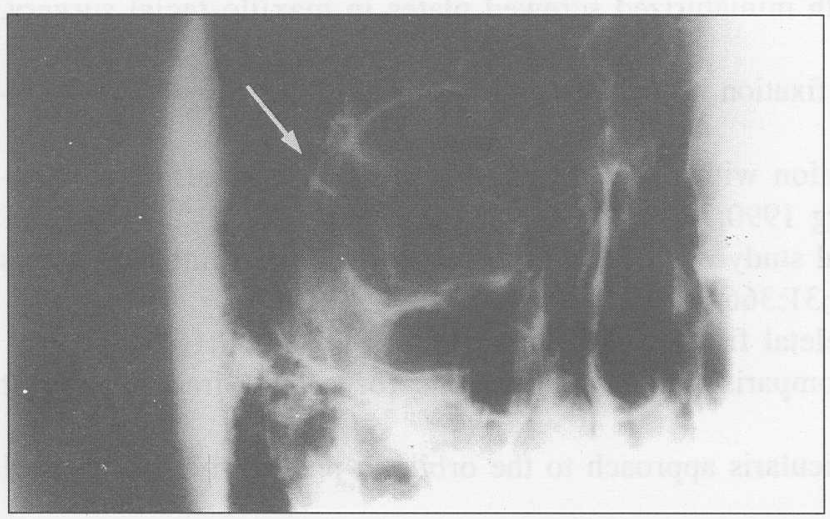

Figure 6. Orbital radiograph showing the mini-plate at the right fronto-zygomatic suture. interincisally. The right zygomatic complex was depressed with step deformity noted on the infraorbital rim.

Radiological examination confirmed a diagnosis of right zygomatic complex fracture, upper right dentoalveolar fracture extending from the lateral incisor tooth to the second molar tooth and fractures of the mandible occurring on the right body near the angle and mesial to the left canine tooth.

A week later, he was taken to theatre for reduction of the facial fractures. The right zygomatic complex fracture was reduced via Gillies' approach and the right fronto-zygomatic suture was plated with the miniplate via the lateral eyebrow incision (Fig. 6). The fracture was stable with a single fixation. The right mandibular fracture was reduced and immobilised with miniplates

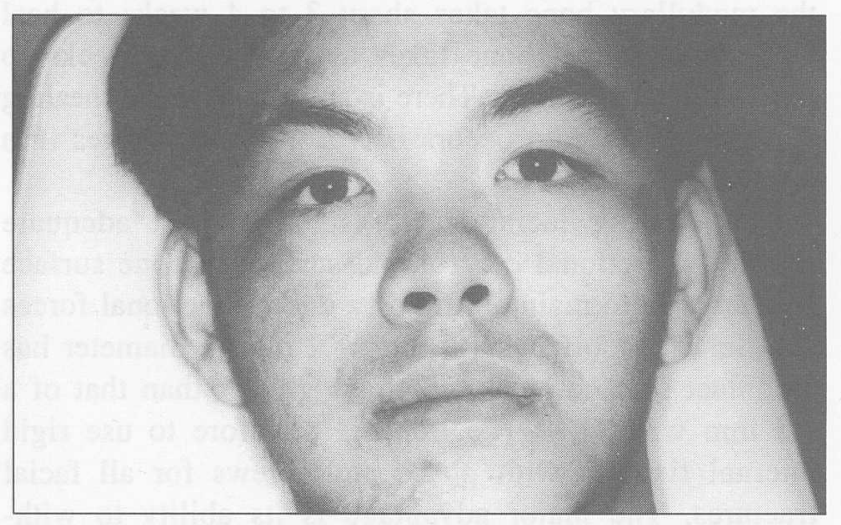

Figure 7. Postoperative appearance six weeks later.

as well. No intermaxillary fixation was done.

Postoperatively, the patient progressed well and was discharged from the hospital on the tenth day. Six weeks later, the upper and lower arch bars were removed (Fig. 7). The patient however complained of discomfort involving a remaining mesial root of the lower right third molar three months after his original injury. This root together with the miniplate that was placed nearby on the mandible were removed surgically under local anaesthesia. There was no complaint of the plated zygomatic fracture. Review of the patient done on the sixth month indicated that healing was uneventful. Two years later this patient was also symptom free with good facial aesthetics and occlusal function.

\section{DISCUSSION}

Management of zygomatic complex fractures depends on a few factors. Surgical intervention is usually not necessary if there is minimal displacement with no significant functional impairment. Studies by Foo (10) showed that approximately $51 \%$ of the cases had no surgical intervention. This percentage is higher than that reported by Ellis et al. (11) and Pospisil et al. (1) where $23 \%$ and $37 \%$ of their cases respectively, were not treated surgically. With surgical intervention, a high percentage was reported by Ellis et al. (70\%) using the Gillies' approach alone. Sometimes, the zygomatic complex fractures are unstable after reduction and will 
require some forms of fixation. Ellis et al. indicated that the type of fixation most commonly used in managing unilateral zygomatico-orbital fractures was by fronto-zygomatic wire only $(29.8 \%)$. The infra-orbital wires alone were used in $12.9 \%$ of their reported cases (1). The type of approach used was related to the site of the fracture sustained.

Good bone healing is optimized by precise anatomic reduction and rigid immobilisation. In primary bone healing, there is direct restoration of lamellar bone while in secondary bone healing, the process includes haematoma, induction, inflammation, soft and hard callus formation and finally followed by remodelling of the woven bone to lamellar bone. Studies have shown that the medullary bone takes about 3 to 4 weeks to heal while the cortical bone takes as long as 16 weeks to repair after fracture (2). There is no primary bone healing if a gap of $0.8 \mathrm{~mm}$ occurs between cortical edges in a fracture (12).

In treating facial fractures, wires lack adequate rigidity, directional control and surface-to-bone surface contact area to maintain rigidity under functional forces (8). Schilli reported that a screw $2 \mathrm{~mm}$ in diameter has a contact surface area of 7 times greater than that of a $0.5 \mathrm{~mm}$ wire (13). The trend is therefore to use rigid internal fixation with plates and screws for all facial fractures. The major advantage is its ability to withstand deformational forces (4). Miniplates are indicated for mid-facial fractures but for special anatomical sites such as the thin bones of the infraorbital and naso-ethmoidal areas, the micro-plates are more suitable following trauma (7).

Surgically, good cosmetic results are achieved using the lower eyelid incision or modified blepharoplasty and the lateral eyebrow incision for management of the zygomatico-orbital fractures. The cosmetic results of blepharoplasty approach are considered to be superior when compared to the results obtained when using the standard infraorbital incision. It is recommended that the infraorbital crease incision be used only for cases where oedema of the lower eyelid still exists or a blepharoplasty incision is contraindicated (11). The main disadvantage of this approach is the noticeable scarring especially for young patients where such a scar may increase in size with growth. The lateral eyebrow incision is also easy to do with minimal morbidity or few complications rather than following the skin creases or wrinkles around the outer aspect of the eye $(14,15)$.

The case reports show that a single form of rigid internal fixation at the fronto-zygomatic or the infraorbital rim region with the plating system can provide adequate stabilisation for these types of fractures. As a first reconstructive step, this procedure should be considered if the fractured zygomatic bone is stable after immobilisation by a single fixation as compared to multiple types of fixation following reduction. Some authors, however advocate radical primary repair because secondary management of complex orbital injuries in major operations is disappointing due to limitation set by the soft tissues (16). But this should only be stressed if the deformity includes the zygomatic complex, the nasoethmoid area and the internal orbit. The failure of primary management of zygomatic complex fracture is probably due to poor diagnosis or inadequate exposure to trauma sites. Clinically, no posttraumatic orbital deformities were observed in the two reported cases. A combined surgical technique using Gillies' approach and a single form of rigid internal fixation can therefore give good cosmetic results and function in the management of uncomplicated zygomaticoorbital complex fractures.

\section{REFERENCES} 1. Ellis E, El-Attar A, Moos KF. An analysis of 2,067 cases of zygomatico-orbital fracture. J Oral Maxillofac
Surg 1985;43:417-28.

2. Jones JK, Van Sickels JE. Rigid fixation: A review of concepts and treatment of fractures. J Oral Surg 1988;65:13-
8.

3. Michelet FX, Deymes J, Dessus B. Osteosynthesis with miniaturized screwed plates in maxillo-facial surgery.
J Maxillofac Surg 1973;1:79-84.

4. Marsh JL. The use of the Wurtzberg system to facilitate fixation in facial osteotomies. Clin Plast Surg 1989;21:49-
60 .

5. Thaller SR, Reavie D, Daniller A. Rigid internal fixation with miniplates and screws: A cost effective technique for treating mandible fractures? Annal Plast Surg 1990;24:469-74.

6. Bahr W, Lessing R. A comparative animal experimental study of differently dimensioned osteosynthesis screws used in the mid-face. Br J Oral Maxillofac Surg 1993;31:366-9.

7. Luhr HG. A micro-system for cranio-maxillofacial skeletal fixation. J Craniomaxfac Surg 1988;16:312-4. 8. Hotlmann B, Wray RC, Little AG. A randomized comparison of four incisions for orbital fractures. Plast
Reconstr Surg 1981;67:731-7.

9. Lacy MF, Pospisil OA. Lower blepharoplasty post-orbicularis approach to the orbit - a prospective study. $\mathrm{Br} \mathrm{J}$
Oral Maxillofac 1987;25:398-401.

10. Foo GC. Maxillo-facial fractures - A retrospective analysis of 285 cases. Med J Malaysia. 1983;38:178-81.

11. Pospisil OA, Fernando TD. Review of the lower blepharoplasty incision as a surgical approach to zygomatic- 
orbital fractures. Br J Oral and Maxillofac Surg 1984;2:261-8.

12. Reitzik M. Bone repair in the mandible: A histologic and biometric comparison between rigid semirigid fixation. J Oral Maxillofac Surg 1983;41:215-8.

13. Schilli W. Compression plate osteosynthesis through the ASIF system. In: Kruger E, Schilli W, eds. Oral and maxillofacial traumatology. Vol 1. Chicago: Quintessence Publishing Company, 1982:349-370.

14. Rowe NL. Fractures of the zygomatic complex and orbit. In: Rowe NL, Williams J1l, eds. Maxillofacial injuries. Vol 1. Churchill Livingstone, 1985:435-537.

15. Davies AS. Traumatic defects of the orbital floor. Br J Oral Surg 1972; 10:133-43.

16. Hammer B, Prein J. Correction of post-traumatic orbital deformities: operative techniques and review of 26 patients. J Craniomaxfac Surg 1995;23:81-90. 